\section{Associação entre a percepção de atributos ambientais e excesso de peso: um estudo realizado em um município de pequeno porte}

\author{
The association between self-rated living \\ environment and excess weight in a small Brazilian \\ town
}

\author{
Asociación entre las características ambientales \\ percibidas y sobrepeso: un estudio realizado en \\ una ciudad pequeña
}

Marília Augusta Sousa Nascimento 1 Daniela Cristina Candela Zucolotto 1 Daniela Saes Sartorelli 1

\footnotetext{
${ }_{1}$ Faculdade de Medicina de Ribeirão Preto, Universidade de São Paulo, Ribeirão Preto, Brasil.

Correspondência D. S. Sartorelli

Faculdade de Medicina de Ribeirão Preto, Universidade de São Paulo.

Av. Bandeirantes 3900

Ribeirão Preto, SP 14049-900, Brasil.

daniss@fmrp.usp.br
}

\begin{abstract}
This population-based cross-sectional study examined the relationship between self-rated living environment and excess body weight in a sample of 216 adults from Itirapuã, São Paulo State, Brazil. Logistic regression adjusted for gender and age showed that people living far from outdoor areas for physical exercise $[O R=2.05$ (95\%CI: 1.15; 3.66)] and primary schools [OR = 1.99 (95\%CI: 1.13; 3.47)] had higher odds of excess weight. Satisfaction with the quality of available supermarkets [OR $=0.14$ (95\% CI: 0.03 ; 0.69) $p=0.02]$, adequate street lighting $[O R=0.37$ (95\%CI: 0.14; 0.96) $p=0.02]$, and pedestrians walking on sidewalks within one's line-of-sight $[O R=0.41$ (95\%CI: $0.18 ; 0.94)$ $p=0.03$ ] were inversely associated with excess weight. The results suggest that small-town individuals' negative perceptions of their living environment are associated with excess weight.
\end{abstract}

Overweight; Body Weight; Environment

\section{Resumo}

O presente estudo investigou a relação entre a percepção de atributos ambientais e a ocorrência de excesso de peso em adultos residentes em Itirapuã, São Paulo, Brasil. Estudo transversal de base populacional conduzido entre 216 adultos. Em modelos de regressão logística ajustados por sexo e idade, verificou-se que os indivíduos que residiam em locais mais distantes de praças ou locais ao ar livre onde pudessem praticar atividade física $[O R=2,05$ (IC95\%: 1,15; 3,66)], e de escolas de Ensino Fundamental [OR = 1,99 (IC95\%: 1,13; $3,47)$, apresentaram maior chance de ocorrência de excesso de peso. A satisfação com a qualidade de supermercados $[O R=0,14$ (IC95\%: 0,03; 0,69) $p=0,02]$, a satisfação com a iluminação noturna [OR = 0,37 (IC95\%: 0,14; 0,96) $p=0$,02] e a possibilidade de visualizar pedestres transitando pelas calçadas [OR =0,41 (IC95\%: 0,18; 0,94) $p=0,03$ ] estavam inversamente associados à ocorrência de excesso de peso. Os dados sugerem que a percepção de indivíduos residentes em municípios de pequeno porte acerca dos atributos ambientais esteja associada com a ocorrência do excesso de peso.

Sobrepeso; Peso Corporal; Meio Ambiente 


\section{Introdução}

A obesidade faz parte do conjunto das doenças crônicas não transmissíveis (DCNT) e está diretamente relacionada com a ocorrência de hipertensão arterial, diabetes tipo 2, doenças cardiovasculares e alguns tipos de cânceres 1 . Segundo os dados da Pesquisa de Orçamentos Familiares (POF) a prevalência do excesso de peso em adultos triplicou no sexo masculino (de $18,5 \%$ para $50,1 \%$ ) e dobrou no sexo feminino (de $28,7 \%$ para $48 \%$ ) entre 1975 e 2009, representando um importante problema de saúde pública no país 2 .

Quando se buscam explicações para o aumento acelerado do excesso de peso na população durante a recente década os esforços devem concentrar-se na identificação dos fatores comportamentais e ambientais envolvidos 3,4,5. Estudos prévios mostram que a população está inserida em um ambiente que promove a má alimentação e hábitos sedentários, refletindo assim em seus comportamentos de saúde e nas taxas de obesidade $6,7,8$. No entanto, identificar os fatores ambientais que interferem na ocorrência de excesso de peso ainda é um desafio, devido às dificuldades de definição e mensuração deste ambiente ${ }^{9,10}$.

O ambiente obesogênico é aquele que pode influenciar negativamente nas escolhas alimentares dos indivíduos por meio do acesso facilitado a estabelecimentos comerciais que vendem em maior quantidade e variedade alimentos de alta densidade energética e a preços acessíveis, quando comparado a alimentos mais saudáveis 8,9,11. Conjuntamente, há os aspectos que desestimulam a prática de atividades físicas como: ausências de locais próprios para a realização de exercícios e recreação (p. ex. parques, praças, ciclovias, campos), trânsito intenso, iluminação, calçadas inadequadas e falta de segurança $9,12,13,14,15$. Este ambiente que tem se tornado predominante, principalmente nas regiões mais urbanizadas do país, é capaz de afetar a saúde da população por modular seus comportamentos e escolhas 16.

Evidências constataram que o padrão de consumo alimentar, nível de atividade física e taxas de obesidade variam de acordo com a área de residência do indivíduo 16,17. Estudos mostraram que indivíduos que relataram insatisfação em relação ao bairro de residência e percepção desfavorável em relação ao trânsito, segurança, barulho, calçadas e locais para prática de exercícios e recreação apresentaram maior chance de ocorrência de excesso de peso. Vizinhanças mais limpas, arborizadas, com menores taxas de criminalidade, com calçamento adequado, presença de estruturas próprias para recreação e prática de exercícios e maior disponibilidade de estabelecimentos que vendem alimentos saudáveis parecem ter associação inversa com o índice de massa corporal (IMC) 10,17,18,19,20,21,22,23.

Um estudo ecológico, de base populacional, realizado na cidade de São Paulo, Brasil, observou uma associação inversa entre a prevalência de sobrepeso e a densidade de parques e/ou instalações públicas para a prática de atividade física na área de residência dos indivíduos 24 . A relação entre os efeitos do ambiente e desfechos de saúde vêm sendo amplamente investigada em estudos internacionais 12,19,22,25,26,27,28,29. No Brasil, o tema foi previamente explorado em regiões altamente urbanizadas 24,30. Entretanto, investigações conduzidas em áreas menos urbanizadas do país são escassas.

Desta forma, o objetivo do presente estudo foi investigar a relação entre a percepção de atributos ambientais e a ocorrência de excesso de peso em adultos residentes em um município paulista de pequeno porte.

\section{Metodologia}

\section{População e delineamento do estudo}

O presente estudo é do tipo transversal de base populacional em uma amostra representativa de adultos residentes na zona urbana do Município de Itirapuã, São Paulo.

O Município de Itirapuã possui 5.914 habitantes ( $51 \%$ do sexo masculino) e unidade territorial de $161.118 \mathrm{~km}^{2}$. Deste total, $83,3 \%$ dos habitantes residem em área urbana e 16,7\% em área rural. O município está localizado no nordeste do Estado de São Paulo, a 432km da capital paulista.

Para o cálculo do tamanho amostral, os dados da prevalência de obesidade no Brasil em adultos (15\%), segundo dados da POF de 2009 (Instituto Brasileiro de Geografia e Estatística. Informações estatísticas: síntese das informações. http:// www.ibge.gov.br/cidadesat/topwindow.htm?1, acessado em 07/Mar/2012), foram empregados. O cálculo se deu por meio da fórmula 31:

$$
n=\frac{z_{\alpha / 2}^{2} P(1-P)}{\varepsilon^{2}},
$$

na qual o $\mathrm{P}$ representa a prevalência do desfecho, $z_{\alpha / 2}$ o nível de significância adotado e o $\mathcal{E}$ é o erro tolerável de amostragem.

Assim, considerando uma prevalência de $15 \%$, o erro tolerável de amostragem de $5 \%$ e um nível de significância de 5\%, o cálculo do tamanho amostral para estimar a prevalência desejada, foi: 
$n=\frac{1,96^{2} \times 0,15 \times 0,85}{0,05^{2}}=196$.

Acrescentando uma taxa de não resposta equivalente a $10 \%$, temos que o tamanho amostral necessário neste estudo era de 216 pessoas.

Foi realizado um sorteio por conglomerados em primeiro estágio, considerando que cada quarteirão é um conglomerado. Em cada quarteirão há dez domicílios e que, em cada um destes, residem três pessoas elegíveis.

Foram critérios de inclusão: idade entre 18 e 60 anos, residentes nos domicílios dos quarteirões sorteados. Os critérios de exclusão foram: relato de câncer, soro positivo para AIDS, acamados, insuficiência renal e gestação. A unidade amostral foi o domicílio e a unidade de análise foram adultos residentes nos domicílios dos quarteirões sorteados. Definiu-se como residente do domicílio quem dorme no local. Foram considerados como perdas os indivíduos não localizados em duas tentativas de contato no domicílio.

\section{Avaliação antropométrica e classificação do IMC}

A coleta de dados se deu por meio de visita domiciliar. Os participantes foram pesados em balança digital (modelo MEA 07700 Plenna, São Paulo, Brasil), com precisão de $100 \mathrm{~g}$ e capacidade de $150 \mathrm{~kg}$, sem sapatos, vestindo roupas leves. A estatura foi aferida por meio de fita métrica inextensível fixada na parede, e os indivíduos foram orientados a permanecerem eretos com a cabeça posicionada no plano horizontal e calcanhares unidos.

O IMC foi obtido dividindo-se o peso pela estatura ao quadrado. Foram considerados portadores de sobrepeso os participantes com IMC entre 25 e $29,9 \mathrm{~kg} / \mathrm{m}^{2}$ e portadores de obesidade os que apresentaram IMC $\geq 30 \mathrm{~kg} / \mathrm{m}^{2}$. No presente estudo o excesso de peso foi considerado IMC $\geq 25 \mathrm{~kg} / \mathrm{m}^{2} 1$.

\section{Variáveis sociodemográficas e comportamentais}

Dados sociodemográficos e comportamentais foram obtidos por meio de um questionário estruturado:

- Variáveis demográficas: idade (em anos completos); sexo; cor da pele (autorreferida); bairro de residência; escolaridade dos participantes e do chefe da família.

- Variáveis sociais e econômicas: renda mensal familiar e posse de bens.

O Critério de Classificação Econômica Brasil da Associação Brasileira de Empresas de Pesqui- sa (ABEP) foi empregado para classificação econômica das famílias 32 .

\section{Percepção de atributos ambientais}

A percepção de atributos ambientais foi avaliada por meio de um questionário estruturado e adaptado para Escala de Mobilidade Ativa no Ambiente Comunitário (NESWS BRASIL) 33, desenvolvido por Saelens et al. ${ }^{34}$, previamente adaptado e testado quanto à sua reprodutibilidade em um estudo nacional 33.

Para avaliação da reprodutibilidade do instrumento no Brasil, Malavasi et al. 33 aplicaram o questionário em duplicata em 75 adultos, apresentando resultados satisfatórios. Este questionário foi empregado por Florindo et al. 35 em estudo de base populacional conduzido no Distrito de Ermelino Matarazzo, São Paulo, o qual detectou associações entre a percepção do ambiente e a prática de atividades físicas.

O questionário possui 83 questões, a maior parte com quatro alternativas. São perguntas referentes à densidade residencial, proximidade e percepção de acesso aos estabelecimentos em geral e estruturas sociais, facilidade para caminhar e andar de bicicleta, características das ruas, características dos arredores do bairro, segurança em relação ao tráfego, segurança em relação a crimes e satisfação com o bairro.

As repostas sobre o tempo de caminhada entre residência e os estabelecimentos foram agrupadas em menos de 10 minutos e mais de 10 minutos. As questões referentes à percepção de segurança contra crimes no bairro foram agrupadas em: discordo fortemente, mais ou menos e concordo fortemente. As questões sobre a satisfação com o bairro, foram agrupadas em: insatisfeito, mais ou menos e satisfeito. O agrupamento se deu em decorrência da dificuldade dos indivíduos em relatar as repostas na escala original do questionário, conforme verificado em estudo piloto conduzido na mesma população de estudo.

Na questão “A criminalidade faz com que não seja seguro caminhar durante o dia no meu bairro?", as respostas mais ou menos e concordo foram agrupadas, dado à baixa frequência de participantes que relataram estas opções.

\section{Análises estatísticas}

Diferenças de características dos participantes, segundo estado nutricional, foram investigadas por meio do teste de $\chi^{2}$.

Para avaliar a associação entre a percepção de atributos ambientais e a ocorrência de excesso de peso foram empregados modelos de re- 
gressão logística bivariados ajustados por sexo e idade, considerando-se o excesso de peso como variável dependente. O software SPSS versão 15.0 (SPSS Inc., Chicago, Estados Unidos) foi utilizado nas análises estatísticas. Estabeleceu-se o nível de significância estatística igual ou inferior a 5\% $(\mathrm{p} \leq 0,05)$.

\section{Aspectos éticos}

O presente estudo foi aprovado pelo Comitê de Ética em Pesquisa do Centro de Saúde Escola da Faculdade de Medicina de Ribeirão Preto, Universidade de São Paulo (CEP/CSE - FMRP-USP; Of. no 058, protocolo no 444). Todos os entrevistados assinaram o Termo de Consentimento após a leitura da carta de informação ao participante.

\section{Resultados}

Dentre os 216 adultos avaliados, 55\% eram do sexo feminino, com idade média de 37 anos. Elevada prevalência de sobrepeso (31\%) e obesidade $(25,5 \%)$ foi observada, sendo que apenas dois participantes $(0,9 \%)$ apresentaram baixo peso.

A Tabela 1 descreve as características sociodemográficas dos participantes do estudo. Verificou-se maior proporção de indivíduos na faixa etária entre 18 e 39 anos, casados, com mais de oito anos de estudo e pertencentes à classe social C. Houve diferença de estado civil segundo estado nutricional ( $\mathrm{p}<0,05$, teste $\chi^{2}$ ).

A Tabela 2 apresenta associação entre o tempo de caminhada da residência e os estabelecimentos em geral e estruturas sociais e a presença de excesso de peso. Em modelos de regressão logística ajustados por sexo e idade, verificou-se que os indivíduos que residiam em locais mais distantes de praças ou locais ao ar livre, onde pudessem praticar atividade física, e de escolas de Ensino Fundamental apresentaram maior chance de ocorrência de excesso de peso. Não houve associação do tempo de caminhada entre a residência e creches, comércio de material de construção e roupas, correio, biblioteca, banco, locadora, farmácia e salão de beleza (dados não apresentados).

Em relação à segurança contra crimes no bairro de residência, verificou-se que a satisfação em relação à iluminação noturna, assim como a possibilidade de visualizar pedestres e ciclistas transitando, estavam inversamente associados à ocorrência de excesso de peso, independente de sexo e idade (Tabela 3). Quanto ao nível de satisfação com o bairro, observou-se que a insatisfação com a qualidade e quantidade de supermercados no bairro de residência estava di-
Tabela 1

Características sociodemográficas segundo presença de excesso de peso em adultos de Itirapuã, São Paulo, Brasil, $2011(N=216)$

\begin{tabular}{|c|c|c|}
\hline Características & $\begin{array}{c}\text { Eutrofia } \\
\text { n (\%) }\end{array}$ & $\begin{array}{c}\text { Excesso de peso } \\
n(\%)\end{array}$ \\
\hline \multicolumn{3}{|l|}{ Sexo } \\
\hline Feminino & $47(51,1)$ & $71(57,3)$ \\
\hline Masculino & $45(48,9)$ & $53(42,7)$ \\
\hline \multicolumn{3}{|l|}{ Idade (anos) } \\
\hline 18-39 & $60(65,2)$ & $67(54,0)$ \\
\hline $40-60$ * & $32(34,8)$ & $57(46,0)$ \\
\hline \multicolumn{3}{|l|}{ Estado civil * } \\
\hline Solteiro & $32(34,8)$ & $26(21,0)$ \\
\hline Casado & $57(62,0)$ & $87(70,2)$ \\
\hline Separado & $0(0,0)$ & $7(5,6)$ \\
\hline Viúvo & $3(3,3)$ & $4(3,2)$ \\
\hline \multicolumn{3}{|l|}{ Anos de estudo } \\
\hline $0-3$ & $5(5,4)$ & $7(5,6)$ \\
\hline $4-7$ & $18(19,6)$ & $33(26,6)$ \\
\hline $8-11$ & $41(44,6)$ & $46(37,1)$ \\
\hline 12 ou mais & $28(30,4)$ & $38(30,6)$ \\
\hline \multicolumn{3}{|l|}{ Cor da pele } \\
\hline Branca & $62(67,4)$ & $94(75,8)$ \\
\hline Não branca & $30(32,6)$ & $30(24,2)$ \\
\hline \multicolumn{3}{|l|}{ Socioeconômico } \\
\hline$A$ e $B$ & $30(32,6)$ & $42(33,9)$ \\
\hline C & $50(54,3)$ & $60(48,4)$ \\
\hline$D$ e $E$ & $12(13,0)$ & $22(17,7)$ \\
\hline
\end{tabular}

* $p<0,05$, teste $\chi^{2}$, para diferença entre eutróficos e portadores de excesso de peso.

retamente associada à ocorrência de excesso de peso, independente de sexo e idade (Tabela 4).

\section{Discussão}

O objetivo do presente estudo foi investigar a relação entre a percepção de atributos ambientais e a ocorrência de excesso de peso em adultos residentes em um município paulista de pequeno porte.

Verificou-se que os indivíduos que residiam em locais mais distantes (> 10 minutos) de praças ou locais para a prática de atividade física ao ar livre, assim como de escolas de Ensino Fundamental, apresentaram maior chance de ocorrência de excesso de peso. Em relação à segurança contra crimes no bairro de residência, observou-se que a percepção da iluminação noturna adequada e 
Associação entre o tempo de caminhada entre a residência e estabelecimentos em geral, estruturas sociais e a presença de excesso de peso em adultos de Itirapuã, São Paulo, Brasil, 2011 ( $\mathrm{N}=216$ ).

\begin{tabular}{|c|c|c|}
\hline Variáveis & OR (IC95\%) bivariado * & OR (IC95\%) ajustado ** \\
\hline \multicolumn{3}{|l|}{ Supermercados } \\
\hline Menos de 10 minutos & 1,00 & 1,00 \\
\hline Mais de 10 minutos & $1,97(1,13 ; 3,44)$ & $1,33(0,71 ; 2,50)$ \\
\hline Valor de $\mathrm{p}$ & 0,017 & 0,38 \\
\hline \multicolumn{3}{|l|}{ Feiras } \\
\hline Menos de 10 minutos & 1,00 & 1,00 \\
\hline Mais de 10 minutos & $1,15(0,66 ; 2,00)$ & $1,17(0,67 ; 2,04)$ \\
\hline Valor de $\mathrm{p}$ & 0,61 & 0,59 \\
\hline \multicolumn{3}{|l|}{ Bares } \\
\hline Menos de 10 minutos & 1,00 & 1,00 \\
\hline Mais de 10 minutos & $1,68(0,97 ; 2,91)$ & $1,27(0,72 ; 2,25)$ \\
\hline Valor de $\mathrm{p}$ & 0,06 & 0,41 \\
\hline \multicolumn{3}{|l|}{ Restaurantes } \\
\hline Menos de 10 minutos & 1,00 & 1,00 \\
\hline Mais de 10 minutos & $1,44(0,79 ; 2,63)$ & $1,41(0,76 ; 2,59)$ \\
\hline Valor de $\mathrm{p}$ & 0,23 & 0,27 \\
\hline \multicolumn{3}{|c|}{ Escolas de ensino fundamental } \\
\hline Menos de 10 minutos & 1,00 & 1,00 \\
\hline Mais de 10 minutos & $1,61(0,89 ; 2,89)$ & $1,99(1,13 ; 3,47)$ \\
\hline Valor de $\mathrm{p}$ & 0,11 & 0,01 \\
\hline \multicolumn{3}{|c|}{ Tempo de transporte entre casa/trabalho/escola } \\
\hline Menos de 10 minutos & 1,00 & 1,00 \\
\hline Mais de 10 minutos & $1,23(0,70 ; 2,17)$ & $1,29(0,73 ; 2,29)$ \\
\hline Valor de $\mathrm{p}$ & 0,47 & 0,39 \\
\hline \multicolumn{3}{|l|}{ Pontos de ônibus } \\
\hline Menos de 10 minutos & 1,00 & 1,00 \\
\hline Mais de 10 minutos & $1,71(0,96 ; 3,06)$ & $1,67(0,91 ; 3,08)$ \\
\hline Valor de $\mathrm{p}$ & 0,07 & 0,09 \\
\hline \multicolumn{3}{|c|}{ Praças ou locais ao ar livre para prática de caminhada } \\
\hline Menos de 10 minutos & 1,00 & 1,00 \\
\hline Mais de 10 minutos & $2,77(1,46 ; 5,27)$ & $2,05(1,15 ; 3,66)$ \\
\hline Valor de $\mathrm{p}$ & 0,002 & 0,015 \\
\hline \multicolumn{3}{|l|}{ Academias de ginástica } \\
\hline Menos de 10 minutos & 1,00 & 1,00 \\
\hline Mais de 10 minutos & $1,22(0,66 ; 2,27)$ & $1,13(0,60 ; 2,13)$ \\
\hline Valor de $\mathrm{p}$ & 0,52 & 0,69 \\
\hline
\end{tabular}

* Modelos de regressão logística bivariados;

** Modelos de regressão logística ajustados por sexo e idade.

a possibilidade de visualizar pedestres e ciclistas transitando pelas calçadas e ruas estavam inversamente associadas à ocorrência de excesso de peso. Quanto ao nível de satisfação com o bairro, foi verificado que a insatisfação com a qualidade e quantidade de supermercados estava diretamente associada à ocorrência de excesso de peso, independente de sexo e idade.
As evidências da influência do ambiente no estilo de vida dos indivíduos e, consequentemente no estado nutricional, são crescentes em estudos conduzidos tanto no exterior 12,19,22,25,26,27,28,29 como no Brasil 24,30. Entretanto, o presente estudo foi inédito em investigar a relação entre a percepção de atributos ambientais e o estado nutricional em adultos residentes em uma cidade de pequeno porte no país. 
Tabela 3

Associação entre a percepção dos participantes quanto à segurança contra crimes no bairro e presença de excesso de peso em adultos de Itirapuã, São Paulo, Brasil, $2011(\mathrm{~N}=216)$.

\begin{tabular}{|c|c|c|}
\hline Variáveis & OR (IC95\%) * & OR (IC95\%) ajustado ** \\
\hline \multicolumn{3}{|c|}{ As ruas do meu bairro são bem iluminadas à noite. } \\
\hline Discordo fortemente & 1,00 & 1,00 \\
\hline Mais ou menos & $0,58(0,22 ; 1,51)$ & $0,63(0,24 ; 1,65)$ \\
\hline Concordo fortemente & $0,36(0,14 ; 0,94)$ & $0,37(0,14 ; 0,96)$ \\
\hline Valor de $p$ & 0,02 & 0,02 \\
\hline \multicolumn{3}{|c|}{ Pedestres e ciclistas que utilizam as ruas do meu bairro são facilmente visualizados pelos } \\
\hline \multicolumn{3}{|c|}{ moradores de dentro de suas casas. } \\
\hline Discordo fortemente & 1,00 & 1,00 \\
\hline Mais ou menos & $0,56(0,24 ; 1,32)$ & $0,19(0,23 ; 1,33)$ \\
\hline Concordo fortemente & $0,43(0,19 ; 0,99)$ & $0,41(0,18 ; 0,94)$ \\
\hline Valor de p & 0,05 & 0,03 \\
\hline \multicolumn{3}{|c|}{ A criminalidade faz com que não seja seguro caminhar durante o dia no meu bairro } \\
\hline Discordo & 1,00 & 1,00 \\
\hline Concordo & $1,72(0,51 ; 5,77)$ & $1,88(0,55 ; 6,38)$ \\
\hline Valor de $p$ & 0,38 & 0,31 \\
\hline \multicolumn{3}{|c|}{ A criminalidade faz com que não seja seguro caminhar durante à noite no meu bairro } \\
\hline Discordo fortemente & 1,00 & 1,00 \\
\hline Mais ou menos & $1,07(0,61 ; 1,88)$ & $1,18(0,66 ; 2,09)$ \\
\hline Concordo fortemente & $5,60(0,67 ; 4,67)$ & $6,22(0,73 ; 5,32)$ \\
\hline Valor de $\mathrm{p}$ & 0,25 & 0,15 \\
\hline \multicolumn{3}{|c|}{ Existe um alto índice de criminalidade no meu bairro } \\
\hline Discordo fortemente & 1,00 & 1,00 \\
\hline Mais ou menos & $0,84(0,45 ; 1,55)$ & $0,99(0,52 ; 1,88)$ \\
\hline Concordo fortemente & $0,60(0,20 ; 1,74)$ & $0,54(0,18 ; 1,60)$ \\
\hline Valor de $p$ & 0,31 & 0,41 \\
\hline
\end{tabular}

* Modelos de regressão logística bivariados;

** Modelos de regressão logística ajustados por sexo e idade.

Elevada prevalência de obesidade (25,5\%) foi verificada na população de estudo, sendo superior à prevalência média nacional (15\%) observada no ano de 2009 2. O cálculo inicial do tamanho amostral foi baseado na estimativa da prevalência de obesidade no Brasil, entretanto, dada a discrepância dos resultados, o desfecho de interesse do presente estudo foi alterado para excesso de peso, o que consiste em uma limitação do estudo.

Estudos sugerem que o menor tempo de caminhada entre a residência e locais para a prática de atividade física e equipamentos sociais estejam diretamente associados com a adoção de hábitos de vida saudáveis e, consequentemente, com o peso corporal adequado. Resultados semelhantes ao do presente estudo foram encontrados por Toftager et al. 36 , ao analisarem a percepção de adultos dinamarqueses em rela- ção à distância de suas residências até locais arborizados, adequados para prática de exercícios, como praças e parques. Os indivíduos que moravam a mais de $1 \mathrm{~km}$ de distância desses locais apresentaram maior chance de serem obesos, quando comparados com aqueles que moravam a menos de 300m. Entretanto, a comparação dos resultados é limitada devido à discrepância das características ambientais avaliadas nos distintos estudos. No presente estudo, a densidade de locais para prática de atividade física na região de moradia não foi explorada, a qual esteve inversamente associada com o excesso de peso em estudos prévios 17,18,19,20,21,22,25. Estas associações sugerem que a disponibilidade e a facilidade de acesso a locais para prática de atividade física e recreação favorecem tal prática, auxiliando na manutenção do peso. 
Tabela 4

Associação entre a satisfação com o bairro de residência e a presença de excesso de peso em adultos de Itirapuã, São Paulo, Brasil, 2011 ( $N$ = 216 )

\begin{tabular}{|c|c|c|}
\hline Variáveis & OR (IC95\%) * & OR (IC95\%) ajustado ** \\
\hline \multicolumn{3}{|c|}{ Acesso ao transporte público no seu bairro? } \\
\hline Insatisfação & 1,00 & 1,00 \\
\hline Mais ou menos & $0,66(0,26 ; 1,66)$ & $0,59(0,19 ; 1,86)$ \\
\hline Satisfeito & $0,70(0,29 ; 1,70)$ & $0,46(0,15 ; 1,44)$ \\
\hline Valor de $\mathrm{p}$ & 0,60 & 0,15 \\
\hline \multicolumn{3}{|c|}{ Acesso ao comércio no seu bairro? } \\
\hline Insatisfação & 1,00 & 1,00 \\
\hline Mais ou menos & $0,43(0,11 ; 1,68)$ & $0,41(0,08 ; 2,15)$ \\
\hline Satisfeito & $0,43(0,11 ; 1,69)$ & $0,31(0,06 ; 1,57)$ \\
\hline Valor de $p$ & 0,45 & 0,12 \\
\hline \multicolumn{3}{|c|}{ Facilidade e prazer em andar a pé nele? } \\
\hline Insatisfação & 1,00 & 1,00 \\
\hline Mais ou menos & $0,41(0,08 ; 2,17)$ & $0,37(0,07 ; 2,01)$ \\
\hline Satisfeito & $0,45(0,09 ; 2,32)$ & $0,40(0,08 ; 2,10)$ \\
\hline Valor de $p$ & 0,77 & 0,66 \\
\hline \multicolumn{3}{|c|}{ Facilidade e prazer em andar de bicicleta nele? } \\
\hline Insatisfação & 1,00 & 1,00 \\
\hline Mais ou menos & $0,68(0,26 ; 1,78)$ & $0,83(0,31 ; 2,22)$ \\
\hline Satisfeito & $0,70(0,27 ; 1,79)$ & $0,89(0,33 ; 2,35)$ \\
\hline Valor de $\mathrm{p}$ & 0,61 & 0,95 \\
\hline \multicolumn{3}{|c|}{ Tempo de transporte entre casa-trabalho/escola? } \\
\hline Insatisfação & 1,00 & 1,00 \\
\hline Mais ou menos & $0,68(0,22 ; 2,10)$ & $0,43(0,11 ; 1,68)$ \\
\hline Satisfeito & $0,53(0,17 ; 1,62)$ & $0,41(0,10 ; 1,65)$ \\
\hline Valor de $\mathrm{p}$ & 0,20 & 0,40 \\
\hline \multicolumn{3}{|c|}{ Quantidade e a qualidade dos restaurantes do seu bairro? } \\
\hline Insatisfação & 1,00 & 1,00 \\
\hline Mais ou menos & $0,55(0,24 ; 1,23)$ & $0,53(0,23 ; 1,21)$ \\
\hline Satisfeito & $0,47(0,16 ; 1,32)$ & $0,42(0,14 ; 1,22)$ \\
\hline Valor de $p$ & 0,14 & 0,11 \\
\hline \multicolumn{3}{|c|}{ Quantidade e qualidade dos mercados/supermercados do seu bairro? } \\
\hline Insatisfação & 1,00 & 1,00 \\
\hline Mais ou menos & $0,14(0,03 ; 0,62)$ & $0,12(0,03 ; 0,55)$ \\
\hline Satisfeito & $0,18(0,04 ; 0,84)$ & $0,14(0,03 ; 0,69)$ \\
\hline Valor de $\mathrm{p}$ & 0,03 & 0,02 \\
\hline \multicolumn{3}{|c|}{ Ser um bom lugar para criar crianças/filhos? } \\
\hline Insatisfação & 1,00 & 1,00 \\
\hline Mais ou menos & $1,04(0,06 ; 17,55)$ & $1,72(0,10 ; 30,24)$ \\
\hline Satisfeito & $1,47(0,09 ; 23,91)$ & $2,18(0,13 ; 36,54)$ \\
\hline Valor de $\mathrm{p}$ & 0,28 & 0,39 \\
\hline \multicolumn{3}{|c|}{ Ser um bom lugar pra se viver? } \\
\hline Insatisfação & 1,00 & 1,00 \\
\hline Mais ou menos & $3,43(0,33 ; 3,55)$ & $4,98(0,47 ; 5,33)$ \\
\hline Satisfeito & $4,37(0,44 ; 4,29)$ & $5,79(0,58 ; 5,81)$ \\
\hline Valor de $p$ & 0,20 & 0,23 \\
\hline
\end{tabular}

* Modelos de regressão logística bivariados;

** Modelos de regressão logística ajustados por sexo e idade. 
No presente estudo, indivíduos que relataram maior distância entre a residência e escolas de Ensino Fundamental apresentam maior chance de excesso de peso, o que poderia ser parcialmente explicado pela possibilidade do uso de veículos como forma de locomoção, em detrimento à caminhada, para levar os filhos na escola, tornando-os mais sedentários e predispondo ao ganho de peso excessivo.

Os indivíduos que reportaram residir próximos a supermercados apresentaram menor chance de excesso de peso, entretanto esta associação não foi independente de sexo e idade. Em análises estratificadas por sexo, verificou-se que esta associação ocorreu apenas entre as mulheres $[\mathrm{OR}=1,97$ (IC95\%: 1,13; 3,44) $\mathrm{p}=0,01]$. Na literatura há controvérsias sobre a associação entre a disponibilidade de supermercados na vizinhança e o excesso de peso 26,37,38.

No presente estudo, verificou-se associação inversa entre a percepção da adequação da iluminação noturna e a possibilidade de visualizar os pedestres transitando pela calçada e a

\section{Resumen}

El presente estudio investigó la relación entre las características ambientales percibidas y el sobrepeso en adultos que viven en Itirapuã, Sao Paulo, Brasil. Es un estudio poblacional transversal realizado entre 216 adultos. En los modelos de regresión logística ajustado por edad y sexo, se encontró que los individuos que viven en los lugares más remotos respecto a plazas de los municipios o al aire libre, donde pudieran practicar la actividad física $[O R=2,05$ (IC95\%: 1,15; 3,66) $p=$ $0,02]$, y las escuelas primarias [OR $=1,99$ (IC95\%: 1,13; $3,47) p=0,01]$, mostraron una mayor frecuencia de sobrepeso. La satisfacción con la calidad de los supermercados [OR = 0,14 (IC95\%: 0,03; 0,69) $p=0,02]$, la percepción de la adecuación de la iluminación [OR = 0,37 (IC95\%: 0,14; 0,96) $p=0,02]$ y la posibilidad de ver a los peatones que transitan por las aceras $[O R=0,41$ (IC95\%: 0,18; 0,94) $p=0,03$ ] se asociaron inversamente con la incidencia de sobrepeso. Los datos sugieren que la percepción de los individuos, que viven en pueblos pequeños, sobre las características ambientales se asocia al sobrepeso.

Sobrepeso; Peso Corporal; Ambiente ocorrência de excesso de peso, sugerindo que a sensação de segurança possa estar associada à adoção de um estilo de vida mais saudável, contribuindo na manutenção do peso corporal saudável. Os dados concordam com estudos prévios que verificaram associações inversas entre a percepção de segurança na vizinhança, assim como baixas taxas de criminalidade, e o excesso de peso $10,18,19,23$.

Dentre as limitações do estudo, destaca-se a ausência de dados sobre a prática de atividade física e do consumo alimentar, que são diretamente associados ao estado nutricional. A adoção do IMC como indicador exclusivo do estado nutricional poderá ter subestimado o problema na população de estudo. Além disso, o desenho transversal do estudo impossibilita verificar-se uma relação causal.

Os dados sugerem que a percepção de indivíduos residentes em municípios de pequeno porte acerca dos atributos ambientais esteja associada com a ocorrência do excesso de peso.

\section{Colaboradores}

M. A. S. Nascimento foi responsável pelo desenho do estudo, coleta de dados, análise de dados e elaboração da primeira versão do manuscrito. D. C. C. Zucolotto e D. S. Sartorelli colaboraram no desenho do estudo, análise e interpretação dos dados e revisão final do manuscrito.

\section{Agradecimentos}

Bolsa de mestrado Capes (M. A. S. N.), bolsa de mestrado FAPESP (D. C. C. Z., processo 2011/03558-7), auxílio à pesquisa da Fundação de Apoio ao Ensino, Pesquisa e Assistência do Hospital das Clínicas, Faculdade de Medicina de Ribeirão Preto, Universidade de São Paulo (FAEPA). 
1. World Health Organization. Diet, nutrition and the prevention of chronic diseases. Report of a Joint WHO/FAO Expert Consultation. Geneva: World Health Organization; 2003.

2. Instituto Brasileiro de Geografia e Estatística. Pesquisa de Orçamentos Familiares, 2008-2009: análise da disponibilidade domiciliar de alimentos e do estado nutricional no Brasil. Rio de Janeiro: Instituto Brasileiro de Geografia e Estatística; 2010.

3. Coutinho W. Etiologia da obesidade. Revista da ABESO 2007; VII(30). http://www.abeso.org.br/pa gina/121/etiologia-da-obesidade.shtml.

4. Caballero B. The global epidemic of obesity: an overview. Epidemiol Rev 2007; 29:1-5.

5. Flegal KM, Carroll MD, Kit BK, Ogden CL. Prevalence of obesity and trends in the distribution of body mass index among US adults, 1999-2010. JAMA 2012; 307:483-90.

6. Brownson RC, Hoehner CM, Day K, Forsyth A, Sallis JF. Measuring the built environment for physical activity: state of the science. Am J Prev Med 2009; 36(4 Suppl):S99-123.

7. Booth KM, Pinkston MM, Carlos Poston SW. Obesity and the built environment. J Am Diet Assoc 2005; 105 Suppl 11:S110-7.

8. Swinburn B, Egger G, Raza F. Dissecting obesogenic environments: the development and application of a framework for identifying and prioritising environmental interventions for obesity. Prev Med 1999; 29:563-70

9. Mackenbach JD, Rutter H, Compernolle S, Glonti $\mathrm{K}$, Oppert JM, Charreire H, et al. Obesogenic environments: a systematic review of the association between the physical environment and adult weight status, the SPOTLIGHT Project. BMC Public Health 2014; 14:233.

10. Mujahid MS, Diez Roux AV, Shen M, Gowda D, Sanchez B, Shea S, et al. Relation between neighborhood environments and obesity in the MultiEthnic Study of Atherosclerosis. Am J Epidemiol 2008; 167:1349-57.

11. Giskes K, Van Lenthe F, Avendano-Pabon M, Brug J. A systematic review of environmental factors and obesogenic dietary intakes among adults: are we getting closer to understanding obesogenic environments? Obes Rev 2011; 12:e95-e106.

12. Casagrande SS, Gittelsohn J, Zonderman AB, Evans MK, Gary-Webb TL. Association of walkability with obesity in Baltimore City, Maryland. Am J Public Health 2011; 101 Suppl:S318-24.

13. Humpel N, Owen N, Leslie E. Environmental factors associated with adults' participation in physical activity: a review. Am J Prev Med 2002; 22:18899.

14. Saelens BE, Handy SL. Built environment correlates of walking: a review. Med Sci Sports Exerc 2010; 40:S550-66.

15. Holsten JE. Obesity and the community food environment: a systematic review. Public Health Nutr 2009; 12:397-405.

16. Lytle LA. Measuring the food environment: state of the science. Am J Prev Med 2009; 36(4 Suppl): S134-44.
17. Powell-Wiley TM, Ayers CR, de Lemos JA, Lakoski SG, Vega GL, Grundy S, et al. Relationship between perceptions about neighborhood environment and prevalent obesity: data from the Dallas Heart Study. Obesity (Silver Spring) 2013; 21: E14-21.

18. Catlin TK, Simões EJ, Brownson RC. Environmental and policy factors associated with overweight among adults in Missouri. Am J Health Promot 2003; 17:249-58.

19. Prince SA, Kristjansson EA, Russell K, Billette JM, Sawada MC, Ali A, et al. Relationships between neighborhoods, physical activity, and obesity: a multilevel analysis of a large Canadian City. Obesity (Silver Spring) 2012; 20:2093-100.

20. Bjork J, Albin M, Grhan P, Jacobsson H, Wadbro J, Ostergren P-O, et al. Recreation values of the natural environment in relation to neighborhood satisfaction, physical activity, obesity and wellbeing. J Epidemiol Community Health 2008; 62:e2.

21. Rundle A, Quinn J, Lovasi G, Bader MD, Yousefzadeh P, Weiss C, et al. Associations between body mass index and park proximity, size, cleanliness and recreational facilities. Am J Health Promot 2013; 27:262-9.

22. Giles-Corti B, Macintyre S, Clarkson JP, Pikora T, Donovan RJ. Environmental and lifestyle factors associated with overweight and obesity in Perth, Australia. Am J Health Promot 2003; 18:93-102.

23. Doyle S, Kelly-Schwartz A, Schlossberg M, Stockard J. Active community environments and health: the relationship of walkable and safe communities to individual health. J Am Plan Assoc 2006; 72:19-31.

24. Jaime PC, Duran AC, Sarti FM, Lock K. Investigating environmental determinants of diet, physical activity, and overweight among adults in São Paulo, Brazil. J Urban Health 2011; 88:567-81.

25. Brown BB, Smith KR, Hanson H, Fan JX, KowaleskiJones L, Zick CD. Neighborhood design for walking and biking: physical activity and body mass index. Am J Prev Med 2013; 44:231-8.

26. Black JL, Macinko J, Dixon LB, Fryer GE. Neighborhoods and obesity in New York City. Health Place 2010; 16:489-99.

27. Ball K, Lamb K, Travaglini N, Ellaway A. Street connectivity and obesity in Glasgow, Scotland: impact of age, sex and socioeconomic position. Health Place 2012; 18:1307-13.

28. Van Dyck D, Cerin E, Cardon G, Deforche B, Sallis JF, Owen N, et al. Physical activity as a mediator of the associations between neighborhood walkability and adiposity in Belgian adults. Health Place 2010; 16:952-60.

29. Santana P, Santos R, Nogueira H. The link between local environment and obesity: a multilevel analysis in the Lisbon Metropolitan Area, Portugal. Soc Sci Med 2009; 68:601-9.

30. Carvalho CE, Leite FHL, Abreu DS, Oliveira MA, Scagliusi FB, Martins PA. Factors associated with overweight in children living in the neighbourhoods of an urban area of Brazil. Public Health Nutr 2012; 15:1056-64. 
31. Bolfarine H, Bussab WO. Elementos de amostragem. São Paulo: Universidade de São Paulo; 1994.

32. Associação Brasileira de Empresas de Pesquisa. Critério de classificação econômica Brasil. São Paulo: Associação Brasileira de Empresas de Pesquisa; 2005.

33. Malavasi LM, Duarte MFS, Both J, Reis RS. Escala de mobilidade ativa no ambiente comunitário - NEWS Brasil: retradução e reprodutibilidade. Rev Bras Cineantropom Desempenho Hum 2007; 9:339-50.

34. Saelens BE, Sallis JF, Black JB, Chen D. Neighborhood based differences in physical activity: An environment scale evaluation. Am J Public Health 2003; 93:1552-8.

35. Florindo AA, Salvador EP, Reis RS, Guimarães VV. Percepção do ambiente e prática de atividade física em adultos residentes em região de baixo nível socioeconômico. Rev Saúde Pública 2011; 45:302-10.
36. Toftager M, Ekholm O, Schipperijn J, Stigsdotter U, Bentsen P, Gronbaek M, et al: Distance to green space and physical activity: a Danish national representative survey. J Phys Act Health 2011, 8:741-9.

37. Hutchinson PL, Nicholas Bodor J, Swalm CM, Rice JC, Rose D. Neighbourhood food environments and obesity in southeast Louisiana. Health Place 2012; 18:854-60.

38. Hattori A, An R, Sturm R. Neighborhood food outlets, diet, and obesity among California adults, 2007 and 2009. Prev Chronic Dis 2013; 10:E35.

Recebido em 31/Jan/2014

Versão final reapresentada em 12/Ago/2014

Aprovado em 18/Ago/2014 